\title{
Design, Developing and Construction of an Educational Solar Water Heating System with Phase Change
}

\author{
Hosni I. Abu-Mulaweh*, Donald W. Mueller, Jr. \\ Purdue University at Fort Wayne, Fort Wayne, IN 46805, USA
}

\begin{abstract}
An educational solar water heater with phase change material (PCM) was designed, developed, and constructed for instructional and demonstrative purposes. This interactive solar water heating system experimental apparatus is capable of demonstrating thermal energy storage and heat transfer concepts and principles. The system consists of two simultaneously functioning heat absorbing units. The first is a flat plate solar collector and the other is a heat storage unit consisting of phase change material (paraffin wax). The heat storage unit utilizes small aluminium cylinders (heat exchangers) filled with paraffin wax as the heat storage medium. Water pump is used to circulate the water between the solar collector and the storage unit where the PCM is located. Results indicate that the PCM stored energy, as latent heat, that was absorbed by the solar collector and released to heat the water in the storage tank when half of the hot water was replaced with cold water. Moreover, tests indicated that latent heat storage is more effective than sensible.
\end{abstract}

Keywords: Experimental Apparatus, Solar Water Heater, Phase Change Material, Design.

\section{Introduction}

For the solar water heating systems to be functional, they require two major components. These two components are a solar collector and a thermal energy storage unit. The solar collector collects the solar radiation and heats the water. Both the solar radiation and the residential water heating demands are time dependent and do not match each other. Thermal energy storage is essential in the solar water heating systems. This is because the peak solar radiation is around noon, while the peak demand for hot water is in the early morning or late evening when solar radiation is not available. Thus the need for thermal storage unit that provides energy needs at all times.

Thermal energy can be stored in the form of sensible heat or latent heat. To store the same amount of thermal energy, a much larger quantities of a storage medium are required for sensible heating storage in comparison to latent heating storage. Moreover, when latent heat storage is used to store solar thermal energy, it can increase the thermal storage efficiency. The latent heat thermal energy storage method is suitable for solar water heating because of the capability of storing a large amount of energy as a phase transition at a constant temperature. Phase change materials (PCMs) are latent heat thermal storage materials. They store energy in the process of changing from solid to liquid state. PCMs store heat as sensible before attaining its phase change temperature and then heat storage continues as latent at constant temperature. The use of PCMs as a storage medium in latent heat thermal energy storage systems offers several advantages such as small unit size, high heat storage capacity and isothermal behavior during charging and discharging.

Latent heat storage has been examined extensively in the past two decades [1-3]. However, a few studies have been reported on the thermal performance of the latent heat thermal energy storage systems using PCMs as a storage medium [4-6]. Recently, Fazilati and Alemrajabi [7] reported an experimental study on the enhancement of solar water heater by using phase change material.

An educational solar water heating system with phase change material was designed, developed, and constructed for instructional and demonstrative purposes. This interactive solar water heating system experimental apparatus is capable of demonstrating thermal energy storage and heat transfer

* Corresponding author. Tel.: +260-481-6357

Fax: +260-481-6281; E-mail: mulaweh@ipfw.edu

(C) 2015 International Association for Sharing Knowledge and Sustainability

DOI: $10.5383 /$ ijtee.10.01.007 
concepts and principles. The objective of this paper is to present the design and development aspects of this educational solar water heating system with phase change materials as well as testing and sample results.

\section{System Specifications}

The design process that was employed in this research project is the one outlined by Bejan et al. [8] and Jaluria [9]. The first essential and basic feature of this process is the formulation of the problem statement. The formulation of the design problem statement involves determining the requirements of the system, the given parameters, the design variables, any limitations or constraints, and any additional considerations arising from safety, financial, environmental, or other concerns.

Since the solar water heating system is required to be designed as a demonstration unit, it needs to be portable and should meet the following specifications:

- The system must be able to collect and store solar energy during daylight hours and transfer this energy to the PCM via water during the same operational period. Energy is transferred from the PCM to the water during the evening and following morning hours when solar energy is not readily available.

- The system apparatus must be mobile. It should be capable of transport to/from laboratory environments.

- The system must demonstrate an educational foundation for the thermal laboratory. It must contain visuals to easily display important thermodynamic and heat transfer concepts. The degree of visibility of the apparatus, such as the reservoir and temperature readouts, can vary based on the given parameters and other design variables. A user manual must also be prepared for proper operation and maintenance.

- The solar collector frame is to be fixed at a 45 degree angle.

- Different materials can be chosen as the PCM for the system based on their price, quantity, melting temperature, and other properties.

- Insulation material will be chosen based on its performance, price, and the ease of application.

\section{Experimental Apparatus}

An educational solar water heating system with phase change material, shown in Fig. 1, was designed and developed. The setup consists of a cylindrical tank which holds inside it the PCM capsules, solar flat plate collector, circulating water pump, flow meter, and data logging device. The stainless steel tank has a capacity of 30 gallons.

Located inside the reservoir are six aluminum heat exchangers. Located inside each heat exchanger is $3.2 \mathrm{~kg}$ of paraffin wax (phase change material). All six cores can be removed individually. The cores are held in place using a machined aluminum plate. For testing, a thermocouple will be located inside the tank to record the water temperature.

The Omega data logger and the electrical box which powers all testing equipment are located on the top of the wooden table. The data logger is a 6-channel Omega brand product which can collect and store temperature readings on an internal 4GB SD card. The workspace is large enough to place textbooks or additional testing equipment on. The electrical box connects to a 6-outlet power strip, and it also powers the water pump.

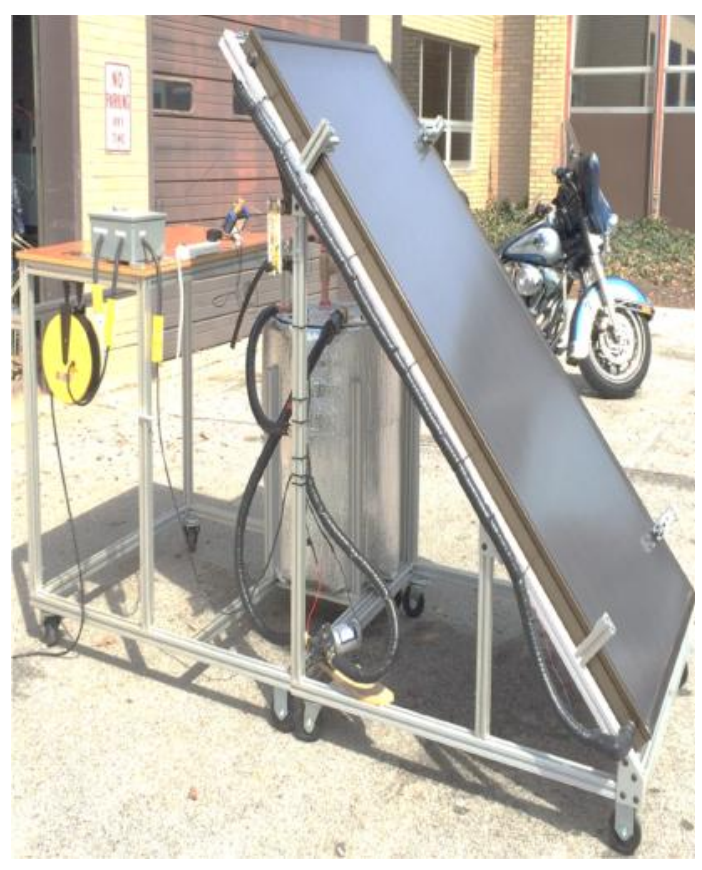

Fig. 1. Solar water heater set up

Figure 2 illustrates the water flow cycle within the system piping loops. The water is pumped out of the bottom of the tank through the water pump. The water travels from the pump through a flow reducing meter which can regulate the flow rate of the system. The water travels into the solar collector, exits the solar collector, and flows back into the water reservoir via the three-way ball valve. The three-way ball valve regulates the path of the water, either back into the reservoir or discharged from the system.

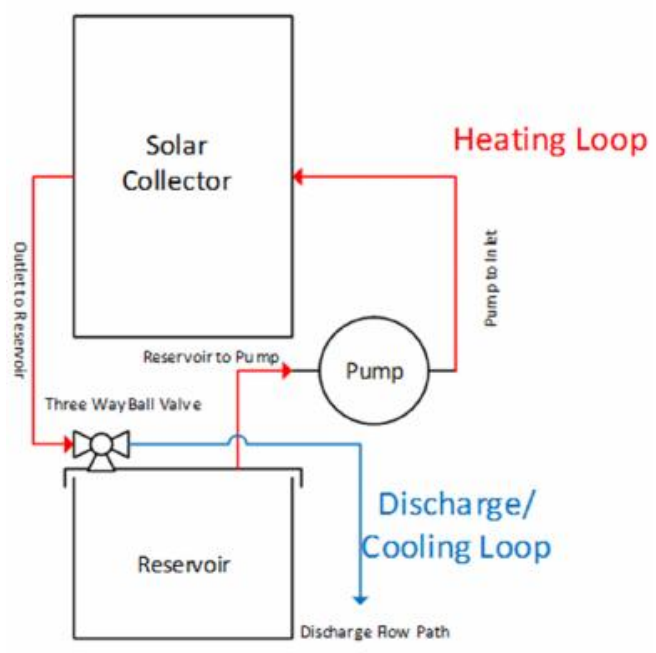

Fig. 2. Water flow cycle within the system piping 


\subsection{Phase Change Materials}

The main objective of the project is incorporating a phase change material. Phase change materials can store a significantly higher quantity of latent heat than non-PCMs. When incorporated into a solar water heating system, a phase change material can store the thermal energy collected from the solar panel and maintain water temperature. During the conceptual design evaluation, paraffin wax was chosen due to its relatively low cost, reliability, and high storage density. It is reported that [10], paraffin wax is taken as the most promising phase change material because it has large latent heat and proper thermal characteristics such as: varied phase change temperature to allow the system to operate in different temperature range, low vapor pressure in the liquid phase, and good thermal and chemical stability.

\subsection{Water Reservoir}

A 30-gallon steel drum was selected as the water reservoir. The drum was modified to fit the design. The top of the drum has two existing threaded holes. These holes were integrated to fit the water inlet (A) and outlet (B). Two additional holes were cut through the lid to be used for water level identification (C) and a fill/relief valve port (D), as shown in Fig. 3. The side of the reservoir was wrapped with a single layer of $3 \frac{1}{2}$ " fiberglass insulation. A final, single-layer of aluminum foil bubble insulation was installed over the fiberglass insulation. Both layers of insulation were cut and taped to avoid interference with the reservoir lid.

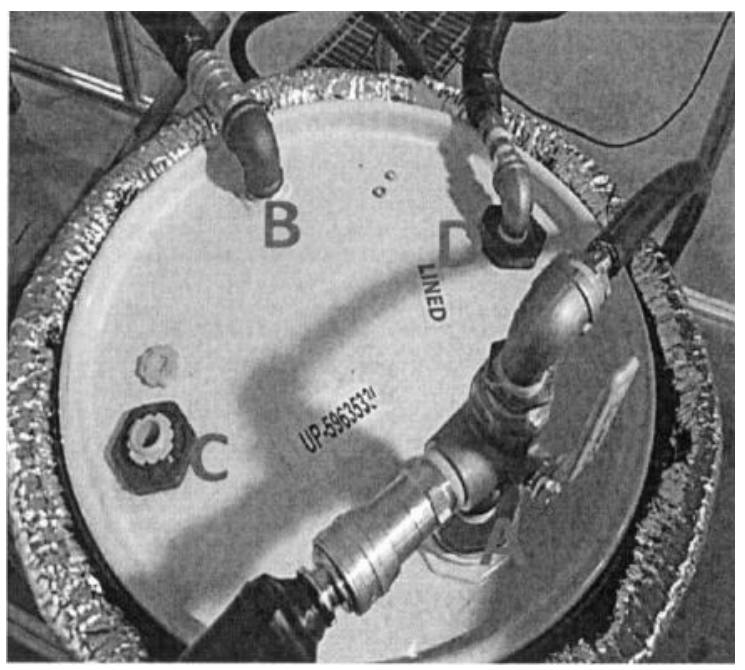

Fig. 3. Water Reservoir Lid

\subsection{Paraffin Wax Heat Exchangers}

To construct the heat exchangers, six 6061-T6 aluminum pipes were purchased and cut to 24 " length. The diameters of the pipes are 4.5" with a wall thickness of 1/4". 6061-T6 Aluminum $1 / 4$ " discs were welded to the bottom and top of each pipe. The heat exchanger tops were drilled and tapped with $1 / 4$ " holes to facilitate the pouring of the paraffin after welding. In order to monitor the temperature in the cores through the melting and solidification processes, type- $T$ thermocouples were placed into the center of the phase change material in two of the cores. To accomplish this while ensuring that the thermocouple stays in the correct location, a thin tube of metal was cut to the correct length and fitted into a second hole that was drilled into two core caps' centers. This thin straw-like tube was then soldered in place and the thermocouples were made to run into the tube 14" where they were fastened with high temperature silicon. Figure 4 shows the six heat exchangers placed inside the water reservoir.

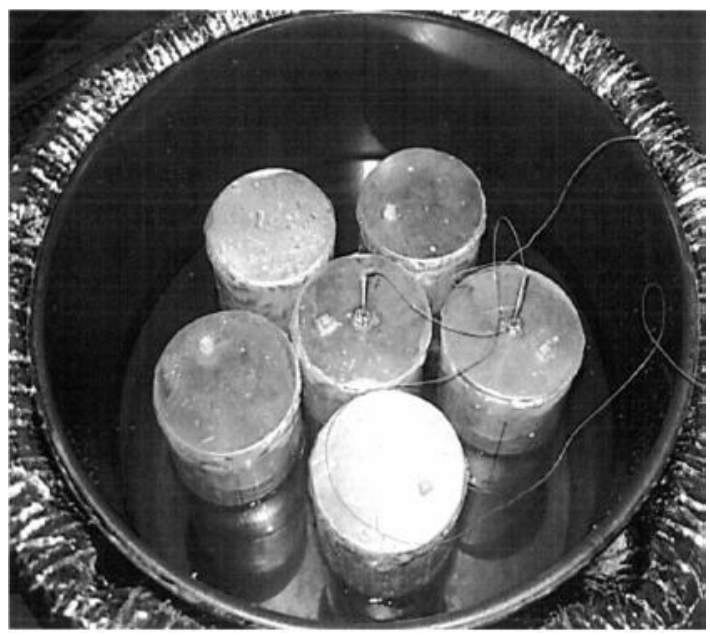

Fig. 4. Heat Exchangers inside the Water Reservoir

\subsection{Data Logger}

Thermocouples were used to measure water temperature in the water reservoir and at the inlet and outlet of solar collector, and ambient air temperature. The data was collected by using an Omega data logger (shown in Fig. 5) that can display and save the temperature measurements. The data is stored on a 4 GB $\mathrm{SD}$ card which can be removed from the data logger and plug it into a lab top to process and analyze the data.

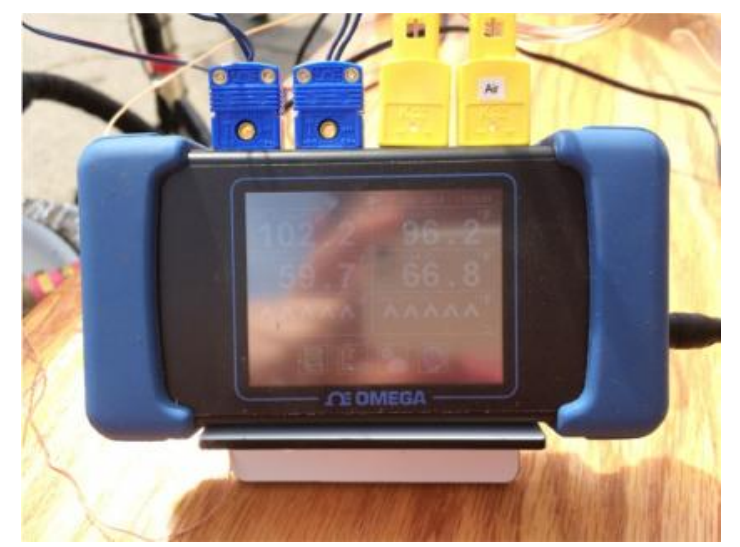

Fig. 5. Data Logger 


\section{Testing and Sample Results}

4.1. Test \#1: 20 Gallons of Water and PCM inside the Reservoir

The six heat exchangers cores filled with the phase change material were placed in the water reservoir along with 20 gallons of water. The system was placed outside for 9 hours during the day while the water pump is running. Before sunset, the water pump was shut off, and the system was stored inside the laboratory. Data collection continued for an additional 15 hours. Fig. 6 shows the water temperature variation inside the water reservoir.

The figure shows that the water temperature inside the reservoir increased from $35.1^{\circ} \mathrm{C}$ at the start of the test to $72.7^{\circ} \mathrm{C}$ by the end of 9 hours heating period. Then the temperature starts to slowly decrease due to heat losses to the ambient.

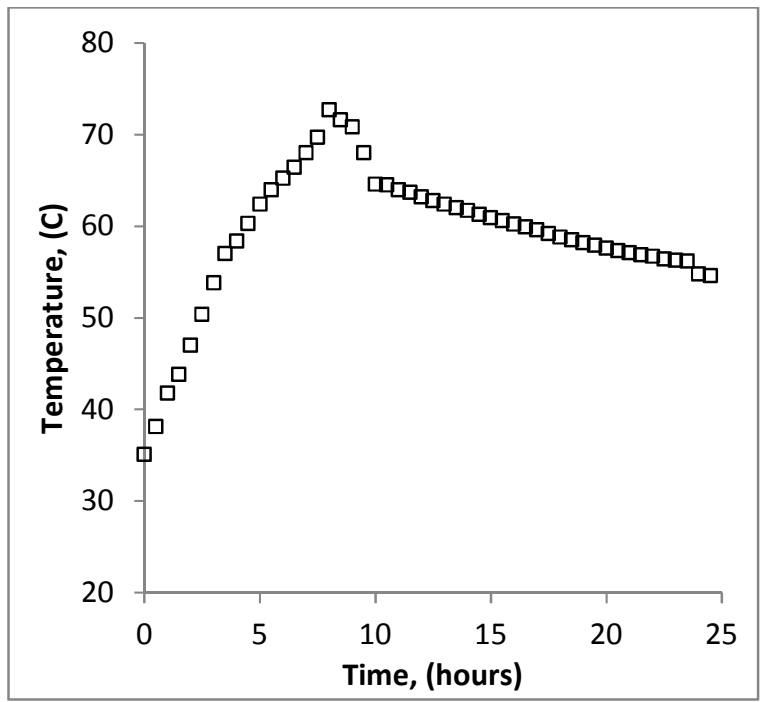

Fig. 6. Water Temperature Variation

\subsection{Test \#2: Replacing 10 Gallons of Hot Water with 10} Gallons of Cold Water and PCM inside the Reservoir

The purpose of this test is to show the effect of PCM material. In this test, the procedure in Test \#1 above was followed. However, at the end of water heating period, 10 gallons of hot water was replaced with 10 gallons of cold water at $19^{\circ} \mathrm{C}$. Just before the 10 gallons water replacement, the hot water temperature was $57.8^{\circ} \mathrm{C}$. Right after the replacement, the temperature hot cold water mixture reached $37.8^{\circ} \mathrm{C}$, as can be seen from Fig. 7. Then the water temperature started to gradually increase and reached $47.1^{\circ} \mathrm{C}$. This increase in temperature is due to the latent heat from the PCM material transferred to the water.

\subsection{Test \#3: Effect of PCM Material}

To show the effect of PCM in heat storage, three different cases were investigated. In the first case, the reservoir was filled with water only. In the second case, the reservoir was filled with water along with $9.5 \mathrm{~kg}$ of aluminium ally. In the third case, the reservoir was filled with water along with the heat exchangers that are filled with the PCM material. For each case, the water in the reservoir was heated using the solar collector as was described in Test \#1 above. In the three cases, 10 gallons of warm water were replaced with 10 gallons of cold water at $19^{\circ} \mathrm{C}$. The water temperature after the replacement is shown in Fig. 8.

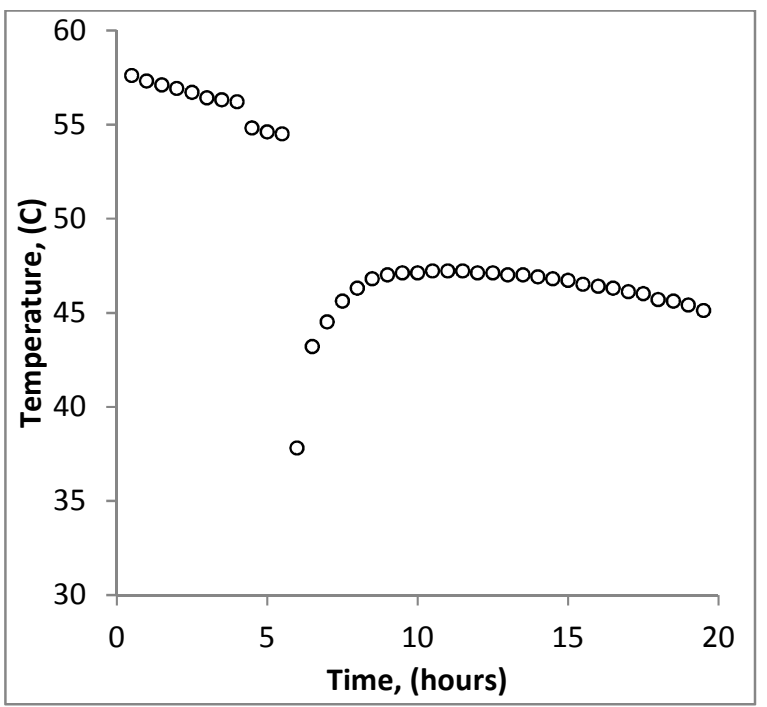

Fig.7. $\quad 10$ Gallons of Hot Water Replaced with 10 Gallons of Cold Water

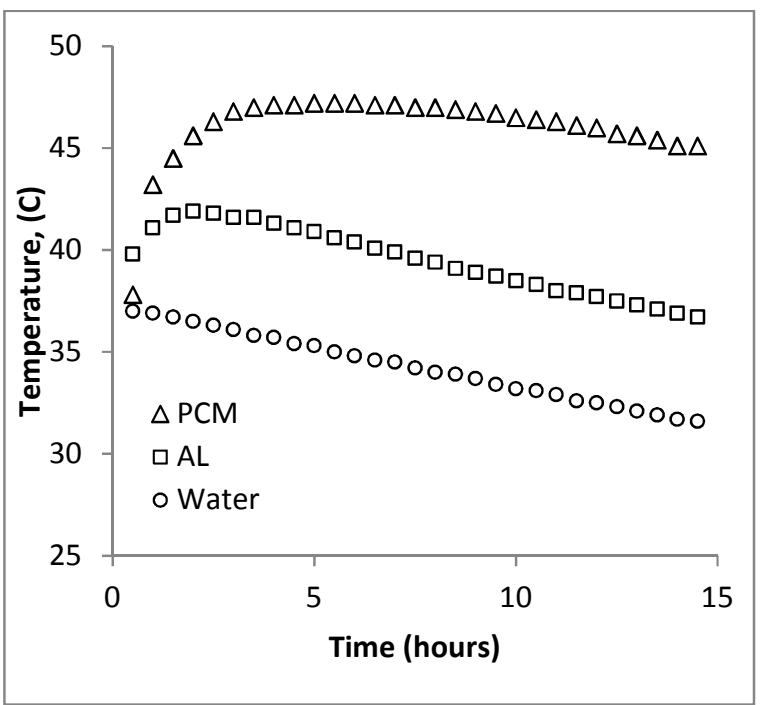

Fig. 8. Effect of PCM Material

\section{Conclusion}

An instructional demonstrative unit of a solar water heater with phase change material (PCM) was designed, developed, and constructed. The solar water heating system experimental apparatus with PCM is capable of demonstrating thermal energy storage and heat transfer concepts and principles. The heat storage unit utilizes small aluminium cylinders (heat 
exchangers) filled with paraffin wax (PCM) as the heat storage medium. Water pump is used to circulate the water between the solar collector and the storage unit where the PCM is located. Results indicate that the PCM stored energy, as latent heat, that was absorbed by the solar collector and released to heat the water in the storage tank when half of the hot water was replaced with cold water. Moreover, tests indicated that latent heat storage is more effective than sensible.

\section{Acknowledgments}

This project was completed with the assistance of an Undergraduate Senior Project Grant from the American Society of Heating, Refrigeration, and Air-Conditioning Engineers (ASHRAE). The members of the Capstone senior design team are Scott Gaskill, Brandon Gratz, Dillon Kelley, and Lei Wang.

\section{References}

[1] Velraj, R., Seeniraj, R.V., Heat transfer studies during solidification of PCM inside an internally finned tube, Journal of Heat Transfer 1999; 121: 493-497.

[2] Velraj, R., Seeniraj, R.V., Hafner, B, Faber, C., Schwarzer, K., Heat transfer enhancement in a latent heat storage system, Solar Energy 1999; 65: 171-180. doi:10.1016/S0038-092X(98)00128-5

[3] Zellba, B, Marin, J.M., Cabeza, L.F., Mehling, H., Review on thermal energy storage with phase change: Materiald, heat transfer analysis and applications, Applied Thermal Engineering 2003; 23: 251-283. doi:10.1016/S1359-4311(02)00192-8
[4] Keumanam, C., Choi, S.H., Thermal characteristics of paraffin in spherical capsul during freeing and melting processes, Int. J. Heat and Mass Transfer 2000; 43: 3183-3196. doi:10.1016/S0017$\underline{9310(99) 00329-4}$

[5] Farid, M.M., Khudhair, A.M., Razack, S.A., AlHallaj, S., A review on phase change energy storage: materials and applications, Energy Conversion and Management 2004; 45: 1597-1615. doi:10.1016/j.enconman.2003.09.015

[6] Mehling, H., Cabeza, L.F., Hippeli, S., Hiebler, S., PCM-module to improve hot water heat stores with stratification, Renewable Energy 2003; 28: 699-711. doi:10.1016/S0960-1481(02)00108-8

[7] Fazilati, M.A., Alemrajabi, A.A., Phase Change material for enhancing solar water heater, an experimental approach, Energy Conversion and Management 2013; 71: 138-145. doi:10.1016/j.enconman.2013.03.034

[8] Bejan, A., Tsatsaronis, G., Moran, M.: Thermal Design \& Optimization, John Wiley \& Sons, Inc., New York, 1996.

[9] Jaluria, Y.: Design and Optimization of Thermal Systems, $2^{\text {nd }}$ Ed., McGraw-Hill, New York, 2007.

[10] Abhat, A., Low temperature latent heat thermal energy storage: heat storage materials, Solar Energy 1983; 30: 313-332. doi:10.1016/0038092X(83)90186-X 